\title{
Optimal Pressure Management for Large-Scale Water Distribution Systems Using Smoothing Model
}

\author{
Pham Duc Dai \\ Thuyloi University No. 175, Tay Son, Dong Da, Hanoi, Vietnam \\ Received: September 08, 2019; Accepted: November 12, 2020
}

\begin{abstract}
Optimal pressure management in water distribution systems (WDSs) is one of the most efficient approaches to control water leakage for water utilities worldwide. The optimal pressure management can be accomplished through regulating operations of pressure reducing valves (PRVs) to ensure that the excessive pressure in the WDS is minimized. This engineering task can be casted into a nonlinear program problem (NLP) with non-smooth constraints. Until now, the non-smooth constraints have been approximated by the smoothing function of Chen Harker-Kanzow-Smale (CHKS). In this paper, instead of using the CHKS function, we propose to apply the uniform smoothing function for formulation of the NLP. Numerical simulations using two smoothing functions will be carried out for optimal pressure managements of a benchmark WDS and a real-world WDS in Thainguyen City, in Vietnam. The comparison results reveal that the NLP formulated with the uniform smoothing function outperforms the existing NLP formulated with the CHKS in terms of optimal solution accuracy.
\end{abstract}

Keywords: Leakage reduction, nonlinear optimization, water distribution systems

\section{Introduction}

Water loss occurs in all water distribution systems (WDSs). The water loss mainly comes from aging, deterioration and inefficient management of WDSs [1]. The method of pressure control is considered as one of the most efficient approaches to decrease water loss and avoid probability of creating new leaks and seepages [2,3]. To control pressure, pressure reducing valves (PRVs) are commonly installed in WDSs to maintain pressures of critical nodes at appropriate values [2, 3]. In general, this engineering problem can be casted into a nonlinear program (NLP) optimization problem where decision variables are pressure settings of PRVs. Vairavamoorthy and Lumbers (1998) [4] formulated a nonlinear programming (NLP) for optimal pressure management to minimize water leakage flow in WDS using sequential quadratic program. Ulanicki et al. 2007 in [5] presented an approach to optimize operation schedules of both boundary and internal PRVs for 24 hours to minimize the leakage flows in domestic meter areas (DMAs) by formulating and solving a NLP. As a result, the control flow modulation curves (FM) for the PRVs, i.e., the relations between the flows and the pressure settings which is used for real-time control of PRV operation, were deduced. Genetic algorithms (GAs) combined with a hydraulic simulator, EPANET 2 [6], were also employed to optimize pressure management of WDSs

Corresponding author: Tel.: (+84) 945047785

Email: daipd@tlu.edu.vn
[7]. De Paola et al. 2017 [8] applied the harmony search algorithm to optimize both PRV locations and PRV pressure settings. Sequential convex programming (SCP), proposed in [9], was used to determine optimal pressure settings of PRVs for optimal pressure managements of DMAs. At first, the method of SCP linearizes the NLP around a feasible NLP solution to a linear program (LP). Then, LP solvers are used to solve the LP to a global solution, which will be used for taking the next linearization. Ghaddar et al. 2017 [10] proposed a new model of PRV for optimal pressure management. The model can only describe two among three operation modes of real PRVs. Thus, it is not appropriate for the case where a PRV works as a check valve to prevent reverse flows.

Non-smooth constraints inherently appear in the formulation of NLP, thus it is impossible to apply standard NLP solvers to solve the NLP. Considering the check valve mode of a PRV, the PRV model becomes non-smooth as discussed in [11]. In addition, bi-directional flows also lead to a kink and non-differentiable problem of the head loss equations for both Hazen-Williams and Darcy-Weisbach formula (i.e., at zero flows). The non-smooth term in the Hazen-Williams head loss equation is smoothed by using the Chen Harker-Kanzow-Smale (CHKS) smoothing functions $[12,13]$. However, the accuracy of these smoothing functions is not properly evaluated.

In this paper, our main contribution is to apply the uniform smoothing function, which is suitable for 
formulation of the NLP model for optimal pressure management of WDSs and outperforms the existing one

\section{Smoothing Nonlinear Program Problem for Optimal Pressure Management}

\subsection{Nonlinear program formulation for optimal pressure regulation}

The objective function to be minimized is defined as the sum of the excessive pressures at all nodes in the WDS in the optimization time horizon $T$ $[1,11]$

$$
\min _{\mathbf{H}, \mathbf{Q}, v} F=\sum_{i=1}^{N J} \sum_{k=1}^{T}\left(H_{i, k}-H_{i, k}^{L}\right)
$$

where $N J$ is the total number of nodes and $T=24$ hours is the time horizon. We consider a WDS with $N P$ pipes, $N R$ reservoirs and NPRV pressure reducing valves (PRVs); $H_{i, k}$ is head at node $i$, at time interval $k ; H_{i, k}^{L}$ is the minimum allowable head at node $i$.

The continuity equation at node $i$

$$
\sum_{j, k} Q_{j, i, k}-d_{i, k}-l_{i, k}=0 ; \quad i=1, \ldots, N J
$$

The leakage amount associated to node $i[2,11]$.

$$
\begin{aligned}
& l_{i, k}=C_{L} L_{t, i} p_{i, k}^{\gamma} \\
& p_{i, k}=H_{i, k}-E_{i} ; L_{t, i}=0.5 \sum_{j} L_{i, j}
\end{aligned}
$$

where $p_{i, k}$ and $E_{i}$ are pressure and elevation of node $i$, respectively; $L_{i, j}$ is length of pipe connecting node $i$ to node $j ; C_{L}$ is given leakage coefficient; $\gamma$ is leakage exponent.

The energy equation for the pipe connecting node $i$ to node $j$

$$
H_{i, k}-H_{j, k}-\Delta H_{i, j, k}=0 ; i j=1, \ldots, N P
$$

where the head loss ( $\left.\Delta H_{i, j}\right)$ is computed by the Hazen-Williams equation [11]

$$
\Delta H_{i, j . k}=\frac{10.67 L_{i, j}}{D_{i, j}^{4.87} C_{i, j}^{1.852}} Q_{i, j, k}\left|Q_{i, j, k}\right|^{0.852}
$$

The energy equation for pressure reducing valve placed in link $i j[10,11]$.

$$
\begin{aligned}
& \max \left(0, H_{i, k}-H_{j, k}\right) \geq R_{i, j} Q_{i, j, k}^{2} \\
& R_{i, j}=\frac{8 K_{i, j}}{\pi^{2} g D_{i, j}^{4}} ; i j=1, \ldots, N P R V
\end{aligned}
$$

Bound constraints for flows and heads are used to ensure the WDS operated properly

$$
\begin{aligned}
& H^{L} \leq H_{i, k} \leq H^{U} ; i=1, \ldots, N J \\
& Q_{i, j}^{L} \leq Q_{i, j, k} \leq Q_{i, j}^{U} ; j=1, \ldots, N P
\end{aligned}
$$

In this paper, the heads of reservoirs are assumed to be constants.

$$
H_{i, k}=\bar{H}_{i} ; \quad i=1, \ldots, N R
$$

The resulting NLP problem has $(N J+N P+N P R V+N R) \times T$ optimization variables. The formulated NLP optimization problem is continuous, but it is non-smooth according to constraints in equations (3), (6), and (9), which are $l_{i, k}=C_{L} L_{t, i} p_{i, k}^{\gamma}, \max \left(0, H_{i, k}-H_{j, k}\right)$, and $\left|Q_{i, j, k}\right|^{0.852}$. The term of $\max \left(0, H_{i, k}-H_{j, k}\right)$ is non-differentiable when $H_{i, k}=H_{j, k}$. The equation $l_{i, k}=C_{L} L_{t, i} p_{i, k}^{\gamma}$ with $p_{i, k}=H_{i, k}-z_{i}$ can cause a numerical problem for NLP algorithms because $p_{i, k}=H_{i, k}-z_{i}<0$ can occur during implementation of NLP algorithms. To overcome this problem, it is necessary to replace $p_{i, k}$ by $\left|H_{i, k}-z_{i}\right|$. Similarly, the term $\left|Q_{i, j, k}\right|^{0.852}$ has a kink at zero flow and non-differentiable. In the next section, we will approximate these non-smooth equations by smooth ones.

\subsection{The existing smoothing function}

In [12] the $\max (0, t)$ can be approximated by

$$
\phi_{C H K S}(t, \mu)=\frac{t+\sqrt{t^{2}+4 \mu^{2}}}{2}
$$

The non-smooth function $|t|$ can be approximated

$$
|t| \simeq \phi_{C H K S}(t, \mu)+\phi_{C H K S}(-t, \mu)=\sqrt{t^{2}+4 \mu^{2}}
$$

This function is commonly used to approximate $|Q|$ in $[5,11]$. These above smoothing functions are known as Chen Harker-Kanzow-Smale (CHKS) smoothing functions in [13]. The parameter $\mu$ is chosen as a small value and that $\lim _{\mu \rightarrow 0} \phi_{C H K S}(t, \mu)=\max (0, t)$. 


\subsection{The uniform smoothing function}

Using the uniform smoothing function, $\max (0, t)$ can be approximated by a strictly and parameter convex function $\phi(t, \mu)$ as [13]

$$
\phi_{U}(t, \mu)= \begin{cases}t & \text { if } t \geq \frac{\mu}{2} \\ 0 & \text { if } t \leq-\frac{\mu}{2} \\ \frac{t^{2}}{2 \mu}+\frac{t}{2}+\frac{\mu}{8} & \text { if }|t|<\frac{\mu}{2}\end{cases}
$$

The derivative of $\phi(t, \mu)$ is

$$
\frac{d \phi_{U}(t, \mu)}{d t}= \begin{cases}1 & \text { if } t \geq \frac{\mu}{2} \\ 0 & \text { if } t \leq-\frac{\mu}{2} \\ \frac{t}{\mu}+\frac{1}{2} & \text { if }|t|<\frac{\mu}{2}\end{cases}
$$

The smoothing function for $|t|$ is

$$
\begin{aligned}
& \psi(t, \mu)=\phi_{U}(t, \mu)+\phi_{U}(-t, \mu) \\
& =\left\{\begin{array}{l}
t \quad \text { if } t \geq \frac{\mu}{2} \\
-t \quad \text { if } t \leq-\frac{\mu}{2} \\
\frac{t^{2}}{\mu}+\frac{\mu}{8} \quad \text { if }|t|<\frac{\mu}{2}
\end{array}\right.
\end{aligned}
$$

And, its derivative is

$$
\frac{d \psi(t, \mu)}{d t}= \begin{cases}1 & \text { if } t \geq \frac{\mu}{2} \\ -1 & \text { if } t \leq-\frac{\mu}{2} \\ \frac{2 t}{\mu} & \text { if }|t|<\frac{\mu}{2}\end{cases}
$$

The CHSK smoothing function $\phi_{C H S K}(t, \mu)$ and the uniform smoothing function $\phi_{U}(t, \mu)$ are shown in Fig.1.

It can be seen from the figure that, with the same parameter value $\mu$, the proposed uniform smoothing function is more accurate than the CHSK smoothing function. Therefore, the approximated functions for absolute and max functions in Eq. (7) and (6) in the formulated NLP can be deduced with higher accuracy. Solving such a highly accurate NLP will result in better control strategy than the use of NLP formulated with the CHSK smoothing function. In the next section, numerical experiments for optimal pressure management will be carried out to evaluate the accuracy of the CHSK and uniform smoothing functions.
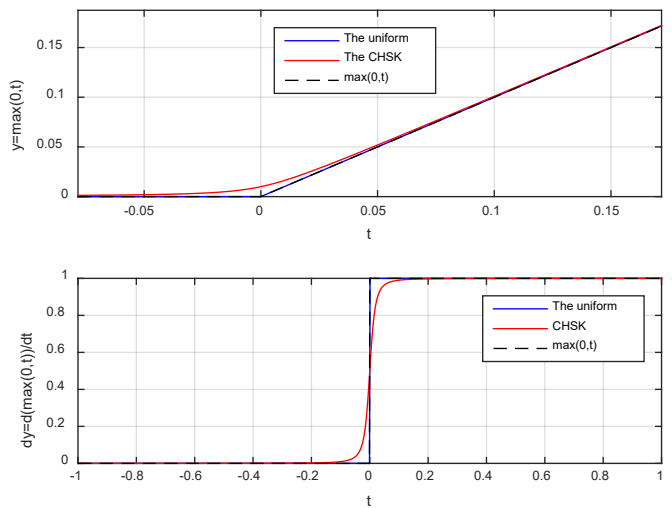

Fig. 1. Comparisons of smoothing functions of max $(0, \mathrm{t})$

\section{Case study}

\subsection{Case study 1}

We consider a benchmark WDS, as shown in Fig.2, comprising of 37 links, 22 nodes, and 3 reservoirs $[2,14]$.

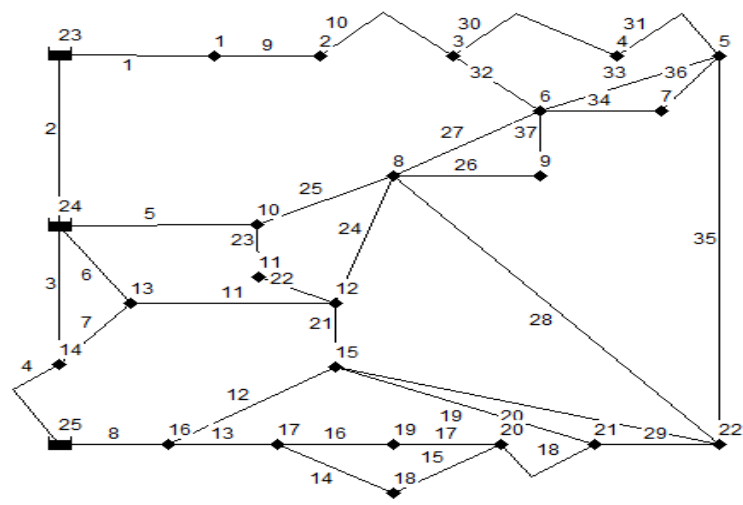

Fig. 2. A benchmark water distribution system

To demonstrate the high accuracy of our proposed smooth model, we compare performances of both formulated nonlinear optimization problems. The first one is our NLP model formulated using the uniform smoothing functions (i.e., using equation (14) to (17)), and the second one is the NLP formulated using the existing smooth function (i.e., equation (12) and (13) ). A nonlinear programming solver, IPOPT in [15], is employed to solve both formulated NLPs. All computation experiments are accomplished on CPU-Pentium (R) Dual-Core 2.8GHz, 3.0GB RAM. Several scenarios on number 
of PRVs installed in WDSs are considered such as: 3 PRVs on link 11, 21, and 20; 4 PRVs on link 1,11,20, and 21; 5 PRVs on link 1,11,20,21, and 29. For each of scenarios, the results of optimal pressure controls from solving both NLPs are applied as inputs for a hydraulic simulator-EPANET 2 [6], the resulting excessive pressure and water leakage amounts are shown in Table 1.

Table 1. Comparisons of NLP solutions

\begin{tabular}{|l|l|l|l|l|}
\hline & \multicolumn{2}{|l|}{ Our new NLP model } & \multicolumn{2}{l|}{ Existing NLP model } \\
\hline$N$ & $\begin{array}{l}\text { OBJ } \\
(\mathrm{m})\end{array}$ & $\begin{array}{l}\text { Leakage } \\
\left(\mathrm{m}^{3} / \text { day }\right)\end{array}$ & $\begin{array}{l}\text { OBJ } \\
(\mathrm{m})\end{array}$ & $\begin{array}{l}\text { Leakage } \\
\left(\mathrm{m}^{3} / \text { day }\right)\end{array}$ \\
\hline 3 & 1462.78 & 2108.16 & 1463.60 & 2109.02 \\
\hline 4 & 982.64 & 2039.90 & 983.40 & 2039.90 \\
\hline 5 & 830.36 & 2020.03 & 835.49 & 2020.89 \\
\hline
\end{tabular}

$O B J$ stands for objective function values; $N$ stands for the number of PRVs installed in the WDS

It can be seen from Table 1 that using our new NLP model, in all scenarios, the obtained results are slightly better than the ones obtained from solving the existing NLP model. In particular, optimal pressure settings of PRVs found by our NLP model lead to lower excessive pressure as well as the amount of water leakage. Fig.3 shows the diurial of water leakage flows for 24 hours for the case of $5 \mathrm{PRVs}$ installed in the WDS (the dotted line).

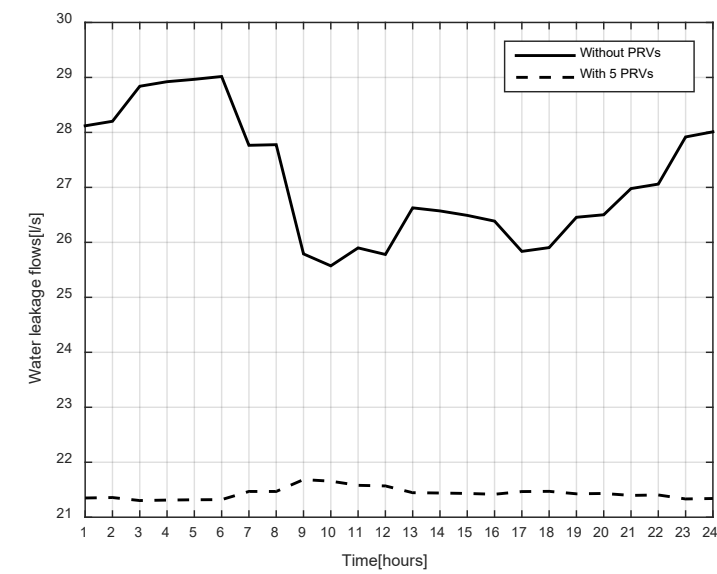

Fig. 3. Comparisons of water leakage flows

Comparing with the case where no pressure control is carried out for the WDS (the thick line), it is seen that optimal pressure management by optimizing pressure settings of PRVs can significantly decrease water leakage amount.

\subsection{Case study 2}

We consider a real world water distribution system in Thainguyen City, in Vietnam as shown in Fig.4. The WDS consists of 112 pipes, 120 nodes, and 4 storage reservoirs. Similar to case study 1 , the performances of two NLPs will be compared in terms of objective function value.

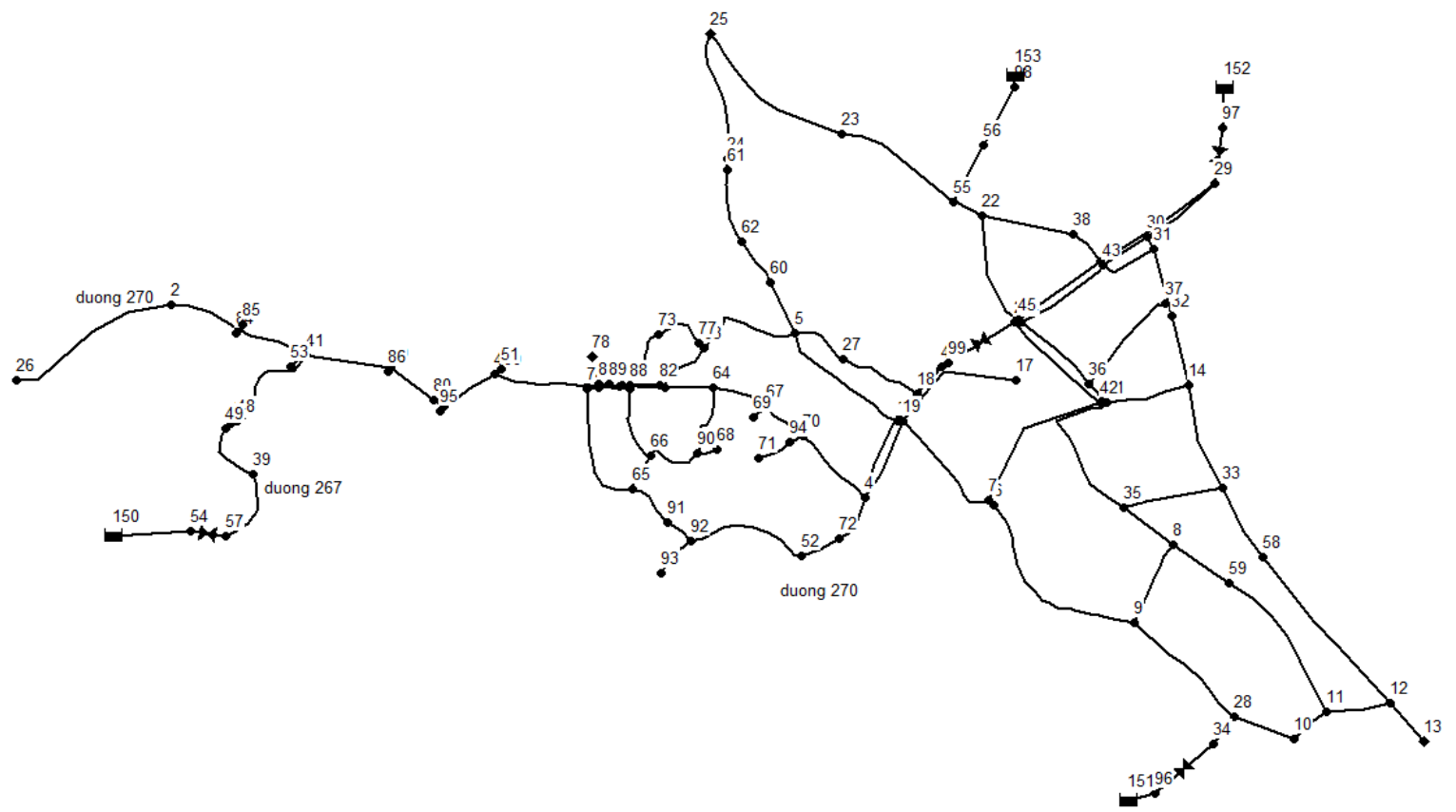

Fig. 4. A real-world WDS in Thainguyen City, Vietnam 


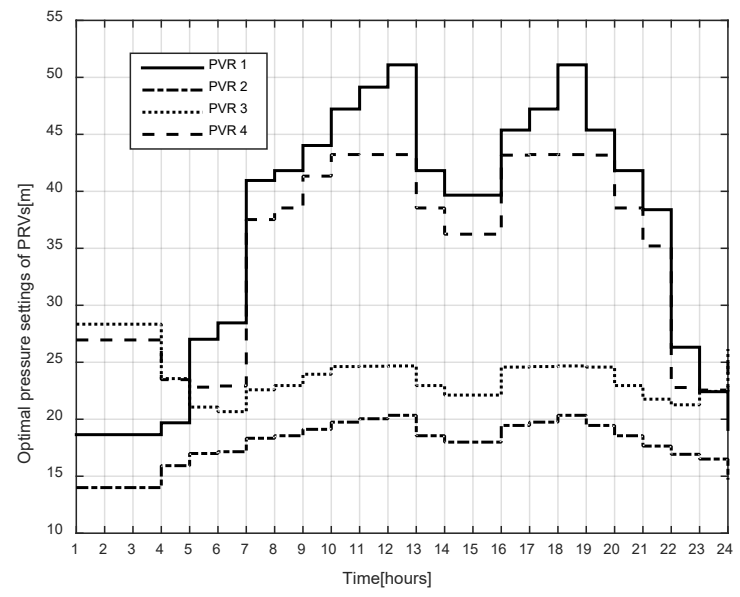

Fig. 5. Optimal pressure settings of PRVs

We consider the case of 4 PRVs installed in the WDS to control pressure. Both NLPs are solved by using IPOPT solver [16]. It took an average computation time of $1.96(\mathrm{~s})$, to solve each of NLPs. The optimal pressure settings for 4 PRVs found by our NLP model are shown in Fig.5. In addition, through simulation, with our smoothing NLP model, the resulting excessive pressure is $27243.73 \mathrm{~m}$, while it is $27372.64 \mathrm{~m}$ by the existing NLP model. It is seen that with our NLP model, we can further reduce $129.0 \mathrm{~m}$ of excessive pressure. The average excessive pressure of the WDS with 4 PRVs is demonstrated in Fig.6 (the dotted line) together with the average excessive pressure where no pressure is carried out (the thick line).

It is seen from the figure that much more excessive pressure is eliminated when pressure settings of PRVs are optimized. This is important because when a WDS is operated under low excessive pressure, leakage rate, as well as the probability of creating new leaks and seepages, are decreased $[5,11]$.

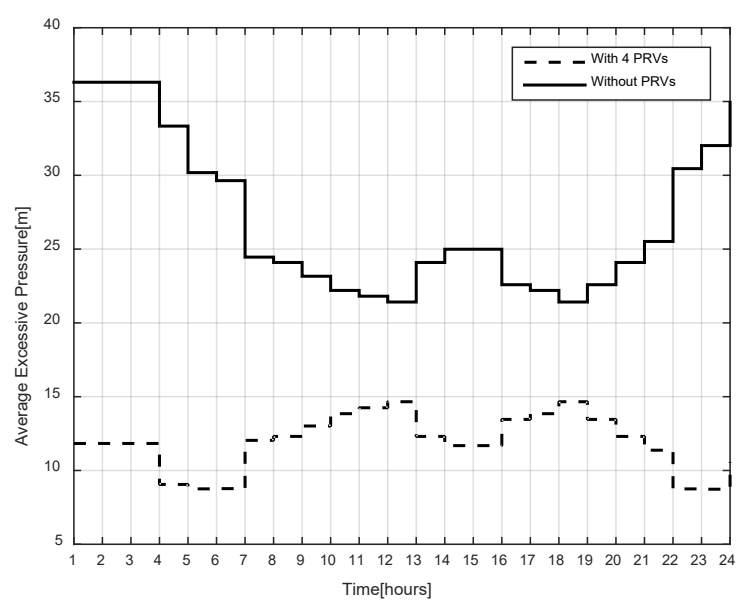

Fig. 6. Comparisons of average excessive pressure

\section{Conclusions}

A highly accurate smoothing NLP model for the problem of optimal pressure management is proposed by using the uniform smoothing function. Numerical simulations have been carried out for optimal pressure regulations for a benchmark WDS and a real-world WDS. The results have shown that using our new NLP model, highly accurate control profiles are obtained leading to lower excessive pressure and water leakage amount. The results of real water leakage amount and excessive pressure are needed to verify in practices and it will be studied in our future research.

\section{References}

[1] Germanopoulos, G., \& Jowitt, P. W.. Leakage Reduction By Excess Pressure Minimization In A Water Supply Network. In ICE Proceedings Vol. 87(2) (1989) 195-214.

[2] Araujo, L. S., Ramos, H., \& Coelho, S. T. Pressure control for leakage minimisation in water distribution systems management. Water resources management, 20(1) (2006) 133-149.

[3] Ulanicki, B., et al. Open and closed loop pressure control for leakage reduction. Urban Water 2(2) (2000) (1998) 105-114

[4] Vairavamoorthy, K., \& Lumbers, J. Leakage reduction in water distribution systems: Optimal valve control. Journal of hydraulic Engineering, 124(11) (1998) 1146-1154.

[5] Ulanicki, B., AbdelMeguid, H., Bounds, P., \& Patel, R.- Pressure control in district metering areas with boundary and internal pressure reducing valves, In Water Distribution Systems Analysis (2008) 1-13.

[6] Rossman, L.A. EPANET2-User's Manual, U.S. Environmental Protection Agency, Cincinnati, Ohio, (2001).

[7] AbdelMeguid, Hossam, and Bogumil Ulanicki, Pressure and leakage management in water distribution systems via flow modulation prvs, Water Distribution Systems Analysis 2010 (2010) 11241139.

[8] De Paola, F., Galdiero, E. and Giugni, M., Location and setting of valves in water distribution networks using a harmony search approach. Journal of Water Resources Planning and Management, 143(6) (2017) 04017015 .

[9] Wright, Robert, et al., Control of water distribution networks with dynamic DMA topology using strictly feasible sequential convex programming, Water Resources Research 51(12) (2015) 9925-9941. 
[10] Ghaddar, B., Claeys, M., Mevissen, M., \& Eck, B. J., Polynomial optimization for water networks: Global solutions for the valve setting problem, European Journal of Operational Research, 261(2) (2017) 450459 .

[11] Dai, Pham Duc, and $\mathrm{Pu} \mathrm{Li}$, Optimal pressure regulation in water distribution systems based on an extended model for pressure reducing valves, Water resources management 30(3) (2016) 1239-1254.

[12] Gopal, Vipin, and Lorenz T. Biegler, Nonsmooth dynamic simulation with linear programming based methods. Computers \& chemical engineering 21(7) (1997) 675-689.
[13] Chen, X., Smoothing methods for nonsmooth, nonconvex minimization. Mathematical programming, 134(1) (2012) 71-99.

[14] Sterling M, Bargiela, A Leakage reduction by optimised control of valves in water networks. Transactions of the Institute of Measurement and Control 6(6) (1984) 293-298

[15] Wächter, A., \& Biegler, L. T., On the implementation of an interior-point filter line-search algorithm for large-scale nonlinear programming. Mathematical programming, 106(1) (2006) 25-57. 